CLNS 03/1841

\title{
A mixing-independent construction of the unitarity triangle
}

\author{
Matthias Neubert \\ F.R. Newman Laboratory for Elementary-Particle Physics \\ Cornell University, Ithaca, NY 14853, USA
}

\begin{abstract}
The study of charmless hadronic two-body decays of $B$ mesons is one of the most fascinating topics in $B$ physics. A construction of the unitarity triangle based on such decays is presented, which is independent of $B-\bar{B}$ and $K-\bar{K}$ mixing. It provides stringent tests of the Standard Model with small theoretical uncertainties.
\end{abstract}

\section{INTRODUCTION}

Measurements of $\left|V_{u b}\right|$ in semileptonic decays, $\left|V_{t d}\right|$ in $B-\bar{B}$ mixing, and $\operatorname{Im}\left(V_{t d}^{2}\right)$ from CP violation in $K-\bar{K}$ and $B-\bar{B}$ mixing have firmly established the existence of a CP-violating phase in the CKM matrix. The present situation, often referred to as the "standard analysis" of the unitarity triangle, is summarized in Figure 1.

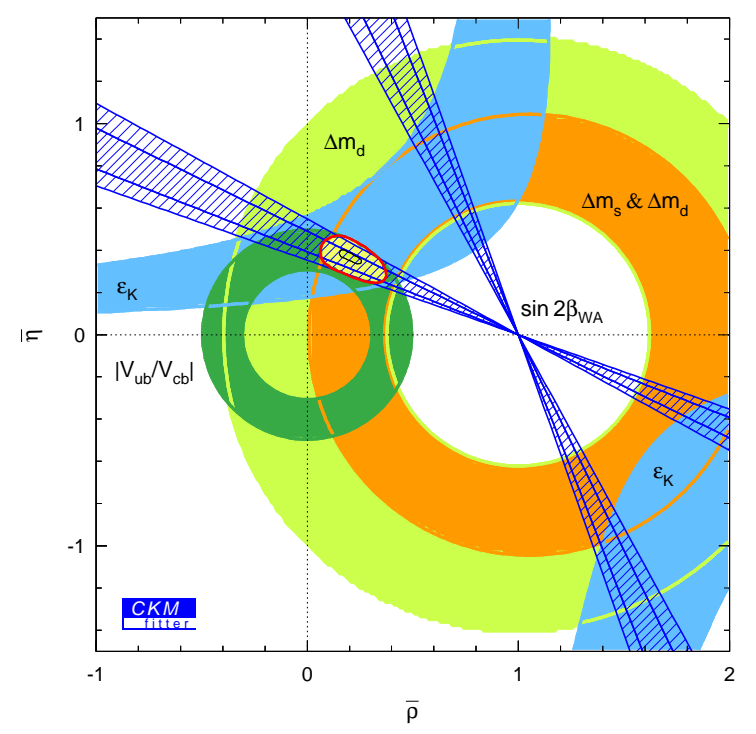

FIGURE 1. Standard constraints on the apex $(\bar{\rho}, \bar{\eta})$ of the unitarity triangle [1].

Three comments are in order concerning this analysis:

1. The measurements of $\mathrm{CP}$ asymmetries in kaon physics $\left(\varepsilon_{K}\right.$ and $\left.\varepsilon^{\prime} / \varepsilon\right)$ and $B-\bar{B}$ mixing $(\sin 2 \beta)$ probe the imaginary part of $V_{t d}$ and so establish CP violation in 
the top sector of the CKM matrix. The Standard Model predicts that the imaginary part of $V_{t d}$ is related, by three-generation unitarity, to the imaginary part of $V_{u b}$, and that those two elements are (to an excellent approximation) the only sources of $\mathrm{CP}$ violation in flavor-changing processes. In order to test this prediction one must explore the phase $\gamma=\arg \left(V_{u b}^{*}\right)$ in the bottom sector of the CKM matrix.

2. With the exception of the $\sin 2 \beta$ measurement the standard analysis is limited by large theoretical uncertainties, which dominate the widths of the various bands in the figure. These uncertainties enter via the calculation of hadronic matrix elements. Below I will discuss some novel methods to constrain the unitarity triangle using charmless hadronic $B$ decays, which are afflicted by smaller hadronic uncertainties and hence provide powerful new tests of the Standard Model, which can complement the standard analysis.

3. With the exception of the measurement of $\left|V_{u b}\right|$ the standard constraints are sensitive to meson-antimeson mixing. Mixing amplitudes are of second order in weak interactions and hence might be most susceptible to effects from physics beyond the Standard Model. The new constraints on $(\bar{\rho}, \bar{\eta})$ discussed below allow a construction of the unitarity triangle that is over-constrained and independent of $B-\bar{B}$ and $K-\bar{K}$ mixing. It is in this sense complementary to the standard analysis.

The phase $\gamma$ can be probed via tree-penguin interference in decays such as $B \rightarrow$ $\pi K, \pi \pi$. Experiment shows that amplitude interference is sizable in these decays. Information about $\gamma$ can be obtained from measurements of direct $\mathrm{CP}$ asymmetries $(\sim \sin \gamma)$, but also from the study of CP-averaged branching fractions $(\sim \cos \gamma)$. The challenge is, of course, to gain theoretical control over the hadronic physics entering the tree-topenguin ratios in the various decays. Recently, much progress has been made toward achieving that goal.

Hadronic weak decays simplify greatly in the heavy-quark limit $m_{b} \gg \Lambda_{\mathrm{QCD}}$. The underlying physics is that a fast-moving light meson produced by a point-like source (the effective weak Hamiltonian) decouples from soft QCD interactions [2, 3]. A systematic implementation of this color transparency argument is provided by the QCD factorization approach $[4,5]$, which makes rigorous predictions for hadronic $B$-decay amplitudes in the heavy-quark limit. One can hardly overemphasize the importance of controlling nonleptonic decay amplitudes in the heavy-quark limit. While a few years ago reliable calculations of such amplitudes appeared to be out of reach, we are now in a situation where hadronic uncertainties enter only at the level of power corrections suppressed by the heavy $b$-quark mass.

In recent work, QCD factorization has been applied to the entire set of the 96 decays of $B$ and $B_{S}$ mesons into $P P$ or $P V$ final states $(P=$ pseudoscalar meson, $V=$ vector meson) [6]. It has been demonstrated that the approach correctly reproduces the main features seen in the data, such as the magnitudes of the various tree and penguin amplitudes, and the fact that they have small relative strong-interaction phases. In the future, when more data become available, this will allow us to extract much useful information about the flavor sector of the Standard Model either from global fits or from analysis of certain classes of decay modes such as $B \rightarrow \pi K, B \rightarrow \pi \pi$, and $B \rightarrow \pi \rho$. Detailed comparison with the data may also reveal limitations of the heavy-quark expansion in certain modes, perhaps hinting at the significance of some power corrections in $\Lambda_{\mathrm{QCD}} / m_{b}$. 

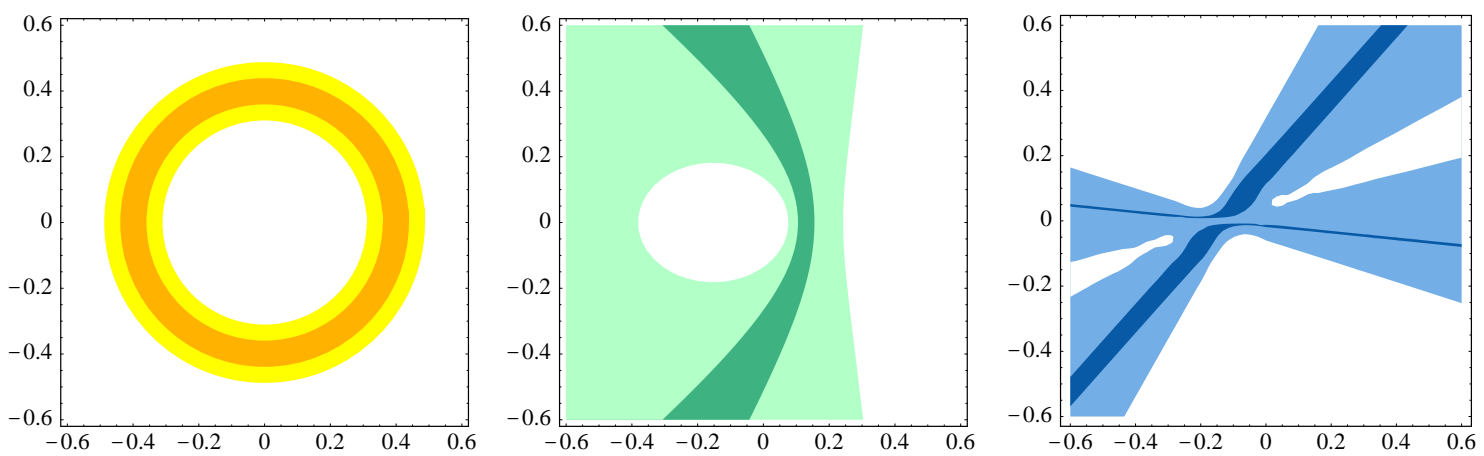

FIGURE 2. The three constraints in the $(\bar{\rho}, \bar{\eta})$ plane used in the construction of the CP- $b$ triangle (see text for explanation). Experimental errors are shown at 95\% CL. In each plot, the dark band shows the theoretical uncertainty, which is much smaller than the experimental error. This demonstrates the great potential of these methods once the data will become more precise.

\section{THE CP-B TRIANGLE}

Despite of the success of QCD factorization in describing the data, there is an interest in analyzing CKM parameters using methods that rely as little as possible on an underlying theoretical framework. In this talk I discuss a method for constructing the unitarity triangle from $B$ physics using measurements whose theoretical interpretation is "clean" in the sense that it only relies on assumptions that can be tested using experimental data. I call this construction the $\mathrm{CP}-b$ triangle, because it probes the existence of a $\mathrm{CP}$ violating phase in the $b$ sector of the CKM matrix. The CP- $b$ triangle is over-determined and can be constructed using already existing data. Most importantly, this construction is insensitive to potential New Physics effects in $B-\bar{B}$ or $K-\bar{K}$ mixing. The present analysis is an update of [7] using the most recent data as of summer 2003.

The first ingredient is the ratio $\left|V_{u b} / V_{c b}\right|$ extracted from semileptonic $B$ decays, whose current value is $\left|V_{u b} / V_{c b}\right|=0.09 \pm 0.02$. Several strategies have been proposed to determine $\left|V_{u b}\right|$ with an accuracy of about $10 \%[8,9,10,11,12]$, which would be a significant improvement. The first plot in Figure 2 shows the corresponding constraint in the $(\bar{\rho}, \bar{\eta})$ plane. Here and below the narrow, dark-colored band shows the theoretical uncertainty, while the lighter band gives the current experimental value.

The second ingredient is a constraint derived from the ratio of the CP-averaged branching fractions for the decays $B^{ \pm} \rightarrow \pi^{ \pm} K_{S}$ and $B^{ \pm} \rightarrow \pi^{0} K^{ \pm}$, using a generalization of the method suggested in [13]. The experimental inputs to this analysis are a certain tree-to-penguin ratio $\varepsilon_{\exp }=0.197 \pm 0.016$ and the ratio

$$
R_{*}=\frac{\operatorname{Br}\left(B^{+} \rightarrow \pi^{+} K^{0}\right)+\operatorname{Br}\left(B^{-} \rightarrow \pi^{-} \bar{K}^{0}\right)}{2\left[\operatorname{Br}\left(B^{+} \rightarrow \pi^{0} K^{+}\right)+\operatorname{Br}\left(B^{-} \rightarrow \pi^{0} K^{-}\right)\right]}=0.804 \pm 0.085
$$

of two CP-averaged $B \rightarrow \pi K$ branching fractions [14]. Without any recourse to QCD factorization this method provides a bound on $\cos \gamma$, which can be turned into a determination of $\cos \gamma$ (for fixed value of $\left|V_{u b}\right| / V_{c b} \mid$ ) when information on the relevant stronginteraction phase $\phi$ is available. The phase $\phi$ is bound by experimental data (and very 
general theoretical arguments) to be small, of order $10^{\circ}$ [7]. (In the future, this phase can be determined directly from the direct $\mathrm{CP}$ asymmetry in $B^{ \pm} \rightarrow \pi^{0} K^{ \pm}$decays.) It is thus conservative to assume that $\cos \phi>0.8$, corresponding to $|\phi|<37^{\circ}$. With this assumption the corresponding allowed region in the $(\bar{\rho}, \bar{\eta})$ plane was analyzed in [5]. The resulting constraint is shown in the second plot in Figure 2.

The third constraint comes from a measurement of the time-dependent $\mathrm{CP}$ asymmetry $S_{\pi \pi}=-\sin 2 \alpha_{\text {eff }}$ in $B \rightarrow \pi^{+} \pi^{-}$decays. The present experimental situation is still unclear, since the measurements by $\operatorname{BaBar}\left(S_{\pi \pi}=-0.40 \pm 0.22 \pm 0.03\right)$ and Belle $\left(S_{\pi \pi}=\right.$ $\left.-1.23 \pm 0.41_{-0.07}^{+0.08}\right)$ are not in good agreement with each other [15]. The naive average of these results gives $S_{\pi \pi}=-0.58 \pm 0.20$. (Inflating the error according to the PDG prescription would yield $S_{\pi \pi}=-0.58 \pm 0.34$, but for some reason the experimenters usually use the naive error without rescaling, and I will follow their example.) The theoretical expression for the asymmetry is

$$
S_{\pi \pi}=-\frac{2 \operatorname{Im} \lambda_{\pi \pi}}{1+\left|\lambda_{\pi \pi}\right|^{2}}, \quad \text { where } \quad \lambda_{\pi \pi}=e^{-i \phi_{d}} \frac{e^{-i \gamma}+(P / T)_{\pi \pi}}{e^{+i \gamma}+(P / T)_{\pi \pi}} .
$$

Here $\phi_{d}$ is the CP-violating phase of the $B_{d}-\bar{B}_{d}$ mixing amplitude, which in the Standard Model equals $2 \beta$. Usually it is argued that for small $(P / T)_{\pi \pi}$ ratio the quantity $\lambda_{\pi \pi}$ is approximately given by $e^{-2 i(\beta+\gamma)}=e^{2 i \alpha}$, and so apart from a "penguin pollution" the asymmetry $S_{\pi \pi} \approx-\sin 2 \alpha$. In order to become insensitive to possible New Physics contributions to the mixing amplitude I adopt a different strategy [5]. I use the measurement $\sin \phi_{d}=0.736 \pm 0.049$ [15] and write $e^{-i \phi_{d}}= \pm\left(1-\sin ^{2} \phi_{d}\right)^{1 / 2}-i \sin \phi_{d}$, with a sign ambiguity in the real part. (The plus sign is suggested by the standard fit of the unitarity triangle.) A measurement of $S_{\pi \pi}$ can then be translated into a constraint on $\gamma$ (or $\bar{\rho}$ and $\bar{\eta}$ ), which remains valid even if the $\sin \phi_{d}$ measurement is affected by New Physics. The result obtained with the current experimental values and assuming $\cos \phi_{d}>0$ is shown in the third plot in Figure 2. The resulting bands for $\cos \phi_{d}<0$ are obtained by a reflection about the $\bar{\rho}$ axis. This follows because the expression for $S_{\pi \pi}$ is invariant under the simultaneous replacements $e^{-i \phi_{d}} \rightarrow-e^{i \phi_{d}}$ and $\gamma \rightarrow-\gamma$.

Each of the three constraints in Figure 2 are, at present, limited by rather large experimental errors, while comparison with Figure 1 shows that the theoretical limitations are smaller than for the standard analysis. Yet, even at the present level of accuracy it is interesting to combine the three constraints and construct the resulting allowed regions for the apex of the unitarity triangle. The result is shown in the left-hand plot in Figure 3. Note that the lines corresponding to the new constraints intersect the circles representing the $\left|V_{u b}\right|$ constraint at large angles, indicating that the three measurements used in the construction of the CP- $b$ triangle provide highly complementary information on $\bar{\rho}$ and $\bar{\eta}$. There are six (partially overlapping) allowed regions, three corresponding to $\cos \phi_{d}>0$ (dark shading) and three to $\cos \phi_{d}<0$ (light shading). If we use the information that the measured value of $\varepsilon_{K}$ requires a positive value of $\bar{\eta}$, then only the solutions in the upper half-plane remain. Comparison with Figure 1 shows that one of these regions (corresponding to $\cos \phi_{d}>0$ ) is in perfect agreement with the standard fit. This is highly nontrivial, since with the exception of $\left|V_{u b}\right|$ none of the standard constraints are used in this construction. Interestingly, there is a second allowed region (corresponding to $\cos \phi_{d}<0$ ) which would be consistent with the constraint from $\varepsilon_{K}$ but inconsistent 

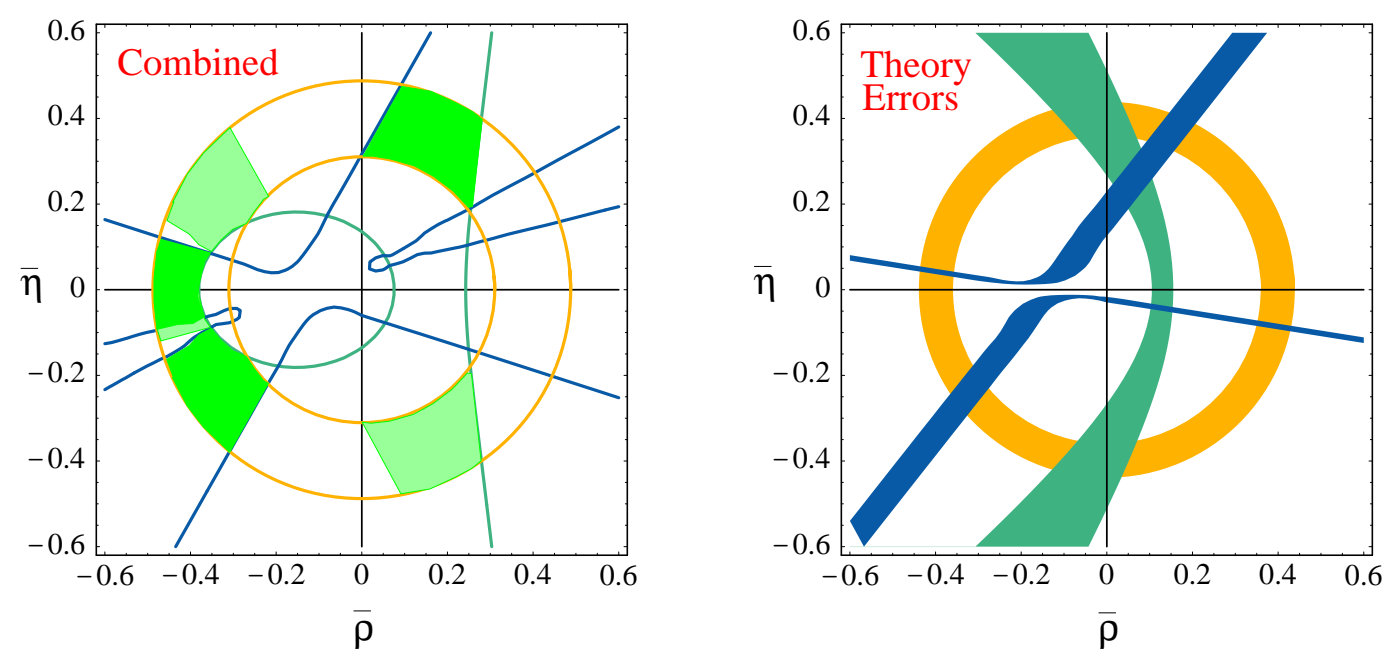

FIGURE 3. Left: Allowed regions in the $(\bar{\rho}, \bar{\eta})$ plane obtained from the construction of the CP- $b$ triangle (at 95\% CL). The light-shaded areas refer to $\cos \phi_{d}<0$. Right: Theoretical error bands for the three constraints combined in the construction of the CP- $b$ triangle.

with the constraints derived from $\sin 2 \beta$ and $\Delta m_{s} / \Delta m_{d}$. Such a solution would require a significant New Physics contribution to $B-\bar{B}$ mixing.

Acknowledgment: This research was supported by the National Science Foundation under Grant PHY-0098631.

\section{REFERENCES}

1. A. Höcker, H. Lacker, S. Laplace and F. Le Diberder, Eur. Phys. J. C 21, 225 (2001); for an updated analysis, see: http://www.slac.stanford.edu/laplace/ckmfitter.html.

2. J. D. Bjorken, Nucl. Phys. Proc. Suppl. 11, 325 (1989).

3. H. D. Politzer and M. B. Wise, Phys. Lett. B 257, 399 (1991).

4. M. Beneke, G. Buchalla, M. Neubert and C. T. Sachrajda, Phys. Rev. Lett. 83, 1914 (1999); Nucl. Phys. B 591, 313 (2000).

5. M. Beneke, G. Buchalla, M. Neubert and C. T. Sachrajda, Nucl. Phys. B 606, 245 (2001).

6. M. Beneke and M. Neubert, Nucl. Phys. B 651, 225 (2003); preprint hep-ph/0308039.

7. M. Neubert, preprint hep-ph/0207327.

8. M. Neubert, Phys. Rev. D 49, 4623 (1994); Phys. Lett. B 543, 269 (2002).

9. R. D. Dikeman and N. G. Uraltsev, Nucl. Phys. B 509, 378 (1998);

I. I. Bigi, R. D. Dikeman and N. Uraltsev, Eur. Phys. J. C 4, 453 (1998).

10. A. F. Falk, Z. Ligeti and M. B. Wise, Phys. Lett. B 406, 225 (1997).

11. C. W. Bauer, Z. Ligeti and M. E. Luke, Phys. Lett. B 479, 395 (2000); Phys. Rev. D 64, 113004 (2001).

12. M. Neubert, JHEP 0007, 022 (2000);

M. Neubert and T. Becher, Phys. Lett. B 535, 127 (2002).

13. M. Neubert and J. L. Rosner, Phys. Lett. B 441, 403 (1998); Phys. Rev. Lett. 81, 5076 (1998); M. Neubert, JHEP 9902, 014 (1999).

14. I use the average of BaBar, Belle, and CLEO data as compiled in the second paper in [6].

15. T. Browder and H. Jawahery, talks presented at the $21^{\text {th }}$ International Symposium on Lepton and Photon Interactions at High Energies, Fermilab, Batavia, Illinois, 11-16 August 2003. 